\title{
ENRICHMENT OF THE DUST-TO-GAS MASS RATIO IN BONDI/JEANS ACCRETION/CLOUD SYSTEMS DUE TO UNEQUAL CHANGES IN DUST AND GAS INCOMING VELOCITIES
}

\author{
P. M. Bellan \\ Applied Physics, California Institute of Technology, Pasadena, CA 91125; pbellan@caltech.edu \\ Received 2006 April 13; accepted 2008 January 23
}

\begin{abstract}
The ratio of the Bondi and Jeans lengths is used to develop a cloud-accretion model that describes both an inner Bondi-type regime where gas pressure is balanced by the gravity of a central star and an outer Jeans-type regime where gas pressure is balanced by gas self-gravity. The gas density profile provided by this model makes a smooth transition from a wind-type inner solution to a Bonnor-Ebert-type outer solution. It is shown that high-velocity dust impinging on this cloud will tend to pile up due to having a different velocity profile from gas so that the dust-to-gas ratio is substantially enriched above the $1 \%$ interstellar medium (ISM) level.
\end{abstract}

Subject headings: accretion, accretion disks - ISM: jets and outflows - MHD

\section{INTRODUCTION}

Laboratory-scale plasma jets have been produced by a magnetohydrodynamic mechanism believed to be analogous to the mechanism responsible for driving astrophysical jets (Hsu \& Bellan 2002; Bellan et al. 2005). The laboratory jets are driven by capacitor bank power supplies that provide poloidal and toroidal magnetic fields, and the jet acceleration mechanism can be considered as due to the pressure of the toroidal magnetic field inflating flux surfaces associated with the poloidal magnetic field. If these jets are indeed related to astrophysical jets, the obvious question arises as to what constitutes the power supply responsible for the toroidal and poloidal magnetic fields in the astrophysical situation. Existing models of astrophysical jets typically assume the poloidal field is simply given and that the toroidal field results from the rotation of an accretion disk twisting up the assumed poloidal field. We have developed an alternate model which postulates that the toroidal and poloidal field result instead from a dusty plasma dynamo mechanism that converts the gravitational energy of infalling dust grains into an electrical power source that drives poloidal and toroidal electric currents creating the respective toroidal and poloidal fields. A brief outline of how infalling charged dust can drive poloidal currents has been presented in Bellan (2007).

An important requirement of the model is that there should be sufficient infalling dust to provide the jet power. It has been well established that the dust-to-gas mass ratio in the interstellar medium (ISM) is $1 \%$. If one assumes, as has been traditional, that this ratio holds throughout the accretion process, then the gravitational energy available from infalling dust would be insufficient. However, Fukue (2001) has recently shown via numerical solution of coupled dust and gas equations of motion that the dust-to-gas ratio can become substantially enriched during Bonditype accretion.

Star formation has also been previously examined from a molecular cloud physics point of view, which differs significantly from the Bondi accretion point of view in the treatment of selfgravity and inflows. Molecular cloud physics is clearly important because observations indicate that stars form within the dense cores of molecular clouds (see, e.g., Evans et al. 2001). Analysis of the force balance in molecular clouds shows that the cloud radial density profile can be characterized by the Bonnor-Ebert sphere solution (Ebert 1955; Bonnor 1956). This solution does not take into account flows associated with accretion. Dust dynamics is also not taken into account; instead it is typically assumed that the dust-to-gas mass ratio is fixed at $1 \%$ for all radii.

To summarize the above discussion, we note that the Bonditype analysis reported by Fukue (2001) emphasizes inflow physics and dust-gas coupling but does not take into account self-gravity, whereas molecular cloud analysis such as used by Evans et al. (2001) emphasizes self-gravity, but does not take into account inflow or dust-gas coupling.

This paper addresses the physics of coupled dust and gas accretion using a methodology similar in concept to that presented by Fukue (2001) but extended to bridge the gap between Bondi accretion models and molecular cloud force balance models. The enrichment mechanism observed by Fukue (2001) is examined in detail and shown to result from the inherently nonuniform nature of the Bondi/molecular cloud/ISM system. Properties of dust and gas for radii ranging from the cloud-ISM interface to the inner Bondi region are considered by examining a sequence of successively smaller concentric regions. The basic character of each region and its scale, defined in terms of the nominal radial distance from a central object star, is as follows:

ISM scale.- The outermost scale is that of the interstellar medium (ISM). The ISM has a gas density $\sim 10^{7} \mathrm{~m}^{-3}$, a dust-togas mass ratio of $1 \%$, and a gas temperature $T_{g}^{\mathrm{ISM}} \sim 100 \mathrm{~K}$, and it is optically thin. The ISM is assumed to be spatially uniform and to bound a molecular cloud having radius $r_{\text {edge }}$.

Molecular cloud scale.- The molecular cloud scale has much higher density than the ISM and is characterized by force balance between gas self-gravity and gas pressure. The molecular cloud scale is subdivided into a large, radially nonuniform, low-density outer region and a small, approximately uniform, high-density inner core region. Clouds have a characteristic scale given by the Jeans length $r_{\mathrm{J}}$. The radial dependence of density is provided by the Bonnor-Ebert sphere solution, which acts as the outer boundary of the Bondi accretion scale.

Bondi accretion scale. - The Bondi accretion scale (Bondi 1952 ) is $\sim r_{\mathrm{B}}$, which is sufficiently small that gas self-gravity no longer matters so equilibrium is instead obtained by force balance between gas pressure and the gravity of a central object assumed to be a star having mass $M_{*}$. The Bondi scale is subdivided into three concentric radial regions: an outermost region where the gas flow is subsonic, a critical transition radius at 
TABLE 1

Sequence of Regions for Gas

\begin{tabular}{clccc}
\hline \hline \multicolumn{1}{c}{ Region } & \multicolumn{1}{c}{ Location } & $\begin{array}{c}n_{g} \\
\left(\mathrm{~m}^{-3}\right)\end{array}$ & $\begin{array}{c}T_{g} \\
(\mathrm{~K})\end{array}$ & $u_{g}$ \\
\hline ISM .............................. & $r>r_{\text {edge }}$ & $10^{7}$ & 100 & $*$ \\
Bonnor-Ebert sphere ......... & $r_{\mathrm{gm}}<r<r_{\text {edge }}$ & $*$ & 10 & $*$ \\
Bondi-Jeans interface........ & $r_{\mathrm{gm}}=\left(r_{\mathrm{B}} r_{\mathrm{J}}\right)^{1 / 2}$ & $*$ & 10 & $*$ \\
Bondi subsonic ................. & $r_{\mathrm{B}}<r<r_{\mathrm{gm}}$ & $*$ & 10 & $<\left(\kappa T_{g} / m_{g}\right)^{1 / 2}$ \\
Bondi transonic ............... & $r=r_{\mathrm{B}}$ & $*$ & 10 & $\left(\kappa T_{g} / m_{g}\right)^{1 / 2}$ \\
Bondi supersonic ............. & $r<r_{\mathrm{B}}$ & $*$ & 10 & $>\left(\kappa T_{g} / m_{g}\right)^{1 / 2}$ \\
\hline
\end{tabular}

NotE.-Asterisks indicate quantities that are discussed/calculated in text.

exactly $r_{\mathrm{B}}$ where the flow is sonic, and an innermost region where the gas flow is free-falling and supersonic.

Collisionless dusty plasma scale (to be considered in a future publication).- - Free-falling dust grains collide with each other in one of the above scales and coagulate to form large-radius grains which are collisionless and optically thin. The optically thin dust absorbs UV photons from the star, photoemits electrons, and becomes electrically charged. The charged dust grains are subject to electromagnetic forces in addition to gravity. Motions of charged dust grains relative to electrons result in electric currents with associated poloidal and toroidal magnetic fields (see preliminary discussion in Bellan 2007).

Jet scale (to be considered in a future publication). - The electric currents interact with the magnetic fields to produce magnetohydrodynamic forces which drive astrophysical jets in a manner analogous to that reported in Hsu \& Bellan (2002) and Bellan et al. (2005).

The separation-of-scales requirement $r_{\mathrm{J}} \gg r_{\mathrm{B}}$ implies existence of a small parameter

$$
\epsilon_{\mathrm{BJ}}=\frac{r_{\mathrm{B}}}{r_{\mathrm{J}}}
$$

quantifying the separation between the Bondi and Jeans scales. For purposes of relating the Bondi and Jeans scales to each other it is convenient to introduce a geometric-mean scale with characteristic length given by

$$
r_{\mathrm{gm}}=\sqrt{r_{\mathrm{B}} r_{\mathrm{J}}}=\sqrt{\epsilon_{\mathrm{BJ}}} r_{\mathrm{J}}
$$

in which case

$$
r_{\mathrm{B}} \ll r_{\mathrm{gm}} \ll r_{\mathrm{J}} .
$$

Gas and dust must be considered separately in each scale. We assume that gas motion influences dust motion but not vice versa so that the dust dynamics can be ascertained after gas dynamics has been determined. This assumption is appropriate so long as dust mass and energy densities are small compared to corresponding gas densities or if the dust is decoupled from the gas. The sequence of scales is summarized in Tables 1 and 2. The numerical values of table elements with asterisks will be predicted by the model presented here, while table elements with given numbers represent prescribed physical boundary conditions. Here $m_{g}$ is the gas molecular mass, $n_{g}$ is the gas density, $T_{g}$ is the gas temperature, $u_{g, d}$ are the radially inward gas and dust fluid velocities, and $\rho_{g, d}$ are the gas and dust mass densities. Quantities with superscripts "ISM" are evaluated in the ISM; unsuperscripted quantities refer to Bondi or molecular cloud regions.

Our methodology is conceptually similar to Fukue's (2001) but also differs in important ways. Fukue (2001) used a wind equation scheme to model Bondi-type physics and, unlike the analysis presented here, assumed the Bondi accretion region was directly bounded by the ISM; i.e., no molecular cloud region with Jeans-type scaling was taken into account. In addition, Fukue assumed that (1) the gas was heated by the combined effects of adiabatic compression and friction due to dust-gas collisions, and (2) the entire region was optically thin so that the dust was subject to radiation pressure. Because of the complexity introduced by the heating of the gas, Fukue obtained results via numerical solution of three coupled differential equations (gas momentum, dust momentum, and gas heating). Our approach differs by assuming that (1) the gas is isothermal, (2) a molecular cloud region lies between the Bondi accretion region and the ISM, and (3) the system is optically thick outside the inner part of the Bondi region so that dust is shielded from optical radiation until it penetrates to the inner part of the Bondi region. Furthermore, rather than using numerical solutions, we attempt analytic solutions as much as possible, a goal made feasible by the isothermal assumption. We believe our assumptions 1-3 reasonably correspond to observations that gas is nearly isothermal and that stars form inside the cores of optically thick molecular clouds.

\section{GAS AND DUST EQUATIONS}

The steady state equation of motion for spherically symmetric gas is

$$
\rho_{g} u_{g} \frac{d u_{g}}{d r}=-\frac{\partial P_{g}}{\partial r}-\rho_{g} \frac{M G}{r^{2}}+f_{\text {drag }} .
$$

Here $u_{g}$ is the radial fluid velocity of the gas, $P_{g}=n_{g} \kappa T_{g}$ is the gas pressure, $\rho_{g}$ is the gas mass density,

$$
M=M_{*}+\int_{r_{*}}^{r} 4 \pi \xi^{2}\left[\rho_{g}(\xi)+\rho_{d}(\xi)\right] d \xi
$$

\begin{tabular}{|c|c|c|c|}
\hline Region & Location & $\rho_{d} / \rho_{g}$ & $u_{d}$ \\
\hline ISM .. & $r>r_{\text {edge }}$ & 0.01 & $3 \mathrm{~km} \mathrm{~s}^{-1}$ (turbulent acceleration) \\
\hline 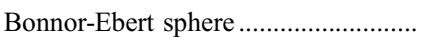 & $\left(r_{\mathrm{B}} r_{\mathrm{J}}\right)^{1 / 2}<r<r_{\text {edge }}$ & $*$ & Slowing down by gas \\
\hline 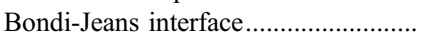 & $r_{\mathrm{gm}}=\left(r_{\mathrm{B}} r_{\mathrm{J}}\right)^{1 / 2}$ & * & Entrained with gas \\
\hline 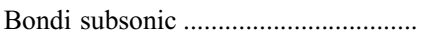 & $r_{\mathrm{B}}<r<r_{\mathrm{gm}}$ & * & Entrained with gas \\
\hline Bondi transonic .......................... & $r=r_{\mathrm{B}}$ & * & Entrained with gas \\
\hline Bondi supersonic & $r<r_{\mathrm{B}}$ & * & Entrained with gas \\
\hline
\end{tabular}

TABLE 2

Sequence of Regions for Dust Physics

Note.-Asterisks indicate quantities that are discussed/calculated in text. 
is the mass inside a sphere of radius $r, \rho_{d}$ is the dust mass density, $M_{*}$ and $r_{*}$ are the mass and radius of the central object star, and $f_{\text {drag }}$ is the drag force on gas due to collisions with dust. Lamers \& Cassinelli (1999, p. 166) give the drag force to be

$$
f_{\text {drag }}=-\left(u_{g}-u_{d}\right) \frac{\rho_{g} \rho_{d}}{m_{d}} \sigma_{d} \sqrt{c_{g}^{2}+\left(u_{d}-u_{g}\right)^{2}} .
$$

The dust behaves as a zero-pressure fluid, so its steady state equation of motion is

$$
\rho_{d} u_{d} \frac{d u_{d}}{d r}=-\rho_{d} \frac{M G}{r^{2}}-f_{\text {drag }}
$$

In the limit that the integral in equation (5) can be ignored, these are the same momentum equations considered by Fukue (2001).

Observations indicate that the gas temperature of clouds, cores, and accretion disks lies in the range $10-30 \mathrm{~K}$ indicating that the gas can be considered approximately isothermal. If the gas were adiabatic, i.e., if $P_{g} \sim \rho_{g}^{5 / 3}$, then the gas temperature would vary as $T_{g} \sim n^{2 / 3}$. Since there is at least a $10^{3}$ increase in gas density from the cloud edge to the Bondi region inner free-fall region, an adiabatically compressed gas volume element would have its temperature increase at least 100-fold as it moved inward. If, in addition, there were heating of gas due to dust frictional drag, the gas temperature would increase even more. For example, the sound speed in Fukue's Figure 1 increases by a factor of approximately 40 from right to left corresponding to a factor of 1600 increase in temperature. Thus, Fukue's Figure 1 analysis predicts that the gas temperature increases from 100 to $1.6 \times 10^{5} \mathrm{~K}$, a temperature at which the molecular hydrogen not only would have become disassociated but would have turned into plasma. Since the observed gas temperature is not $1.6 \times 10^{5} \mathrm{~K}$ but in fact is clamped at $10-30 \mathrm{~K}$, any heat energy resulting from adiabatic compression or friction must be immediately lost, presumably via molecular line emission. In order to conform to observations we therefore assume the gas is isothermal, an approximation which has the incidental, yet fortuitous, side effect of making an analytic approach to the problem feasible.

Since we are assuming that the dust is a perturbation on the gas, we first solve the gas equation ignoring the dust, and then use gas equation solutions as inputs for the dust equation. This approach is self-consistent so long as the dust is approximately decoupled from the gas or if the dust mass and energy densities are much less than the gas mass and energy densities. The approach fails in situations where the dust is coupled to the gas and attains mass or energy densities comparable to the gas. However, the point at which failure occurs is interesting and can be considered a useful prediction of the model.

We now consider gas dynamics while ignoring the dust in which case the gas equation of motion reduces to

$$
\rho_{g} u_{g} \frac{d u_{g}}{d r}=-c_{g}^{2} \frac{d \rho_{g}}{\partial r}-\frac{\rho_{g} G}{r^{2}}\left[M_{*}+\int_{r_{*}}^{r} 4 \pi \xi^{2} \rho_{g}(\xi) d \xi\right],
$$

where

$$
c_{g}=\sqrt{\frac{\kappa T_{g}}{m_{g}}}
$$

is the gas thermal velocity.
Equation (8) has two regimes of interest, namely, (1) an inner regime where $r$ is so small that $M_{*} \gg \int_{r}^{r} 4 \pi \xi^{2} \rho_{g}(\xi) d \xi$, and (2) an outer regime where $r$ is so large that $M_{*} \ll \int_{r_{*}}^{r} 4 \pi \xi^{2} \rho_{g}(\xi) d \xi$. The former regime leads to a Bondi accretion situation characterized by the wind equation discussed by Fukue (2001), while the latter leads to a Jeans-scale problem with Bonnor-Ebert spheres as the solution. We examine these regimes separately and then connect them using an asymptotic technique.

Because the configuration is assumed to be steady state, the gas and dust equations of continuity give the respective accretion rates

$$
\begin{aligned}
& \dot{M}_{g}=-4 \pi r^{2} \rho_{g} u_{g}=\text { const. } \\
& \dot{M}_{d}=-4 \pi r^{2} \rho_{d} u_{d}=\text { const. }
\end{aligned}
$$

where $u_{g}$ and $u_{d}$ are negative, corresponding to radial inward motion.

\section{GAS}

\subsection{Bondi Accretion Region}

In this region it is convenient to normalize the velocity to $c_{g}$ and lengths to the Bondi radius $r_{\mathrm{B}}$, defined as

$$
r_{\mathrm{B}}=\frac{M_{*} G}{2 c_{g}^{2}} .
$$

A bar denotes normalized quantities, and to avoid confusing minus signs, the normalized velocity is defined to be positive so

$$
\begin{gathered}
\bar{u}_{g}=\left|u_{g}\right| / c_{g}, \\
\bar{r}=r / r_{\mathrm{B}} .
\end{gathered}
$$

In addition, the gas mass density is normalized to its value at $r_{\mathrm{gm}}$, the geometric mean of the Bondi and Jeans scales (see eq. [2]), i.e.,

$$
\bar{\rho}_{g}=\rho_{g} / \rho_{\mathrm{gm}}
$$

Thus, $\rho_{\mathrm{gm}}$ is the mass density at a radius much larger than the Bondi length, but much smaller than the Jeans length (see eq. [3]). The normalized form of equation (8) in the Bondi accretion region is thus

$$
\bar{u}_{g} \frac{d \bar{u}_{g}}{d \bar{r}}=-\frac{d \ln \bar{\rho}_{g}}{d \bar{r}}-\frac{2}{\bar{r}^{2}} .
$$

The derivative of equation (10) gives

$$
\frac{d \ln \bar{\rho}_{g}}{d \bar{r}}=-\frac{1}{\bar{u}_{g}} \frac{d \bar{u}_{g}}{d \bar{r}}-\frac{2}{\bar{r}} .
$$

Combining equations (16) and (17) gives the isothermal wind equation (Lamers \& Cassinelli 1999, p. 62)

$$
\left(\bar{u}_{g}^{2}-1\right) \frac{d \bar{u}_{g}}{d \bar{r}}=2 \bar{u}_{g}\left(\frac{1}{\bar{r}}-\frac{1}{\bar{r}^{2}}\right) .
$$


Equation (18) gives the condition that $\bar{u}_{g}=1$ must occur when $\bar{r}=1$ in order for $d \bar{u}_{g} / d \bar{r}$ to be nonsingular at $\bar{r}=1$. Equation (18) can be directly integrated to give

$$
\frac{\bar{u}_{g}^{2}}{2}-\ln \bar{u}_{g}=2 \ln \bar{r}+\frac{2}{\bar{r}}+A,
$$

where $A$ is a constant to be determined. The sonic condition $\bar{u}_{g}=1$ at $\bar{r}=1$ gives $A=-3 / 2$ so equation (19) becomes

$$
\bar{u}_{g} \bar{r}^{2}-\exp \left(\frac{\bar{u}_{g}^{2}}{2}-\frac{2}{\bar{r}}+\frac{3}{2}\right)=0 .
$$

Equation (20), the solution to equation (18), is a transcendental expression relating $\bar{u}_{g}$ and $\bar{r}$ and having two distinct roots. One root has $\bar{u}_{g}$ a monotonically increasing function of $\bar{r}$ and one root has $\bar{u}_{g}$ a monotonically decreasing function. Both roots have $\bar{u}_{g}=1$ at $\bar{r}=1$. The root where $\bar{u}_{g}$ is a monotonically increasing function of $\bar{r}$ (subsonic at small $\bar{r}$, supersonic at large $\bar{r}$ ) is relevant to stellar winds, while the monotonically decreasing root (supersonic at small $\bar{r}$, subsonic at large $\bar{r}$ ) is relevant to the accretion problem discussed here and by Fukue. Since $\bar{u}_{g}$ is subsonic at large $\bar{r}$ and supersonic at small $\bar{r}$, equation (19) has the following limiting forms:

$$
\begin{array}{ll}
\ln \bar{u}_{g}=\ln 2-\frac{1}{2} \ln \bar{r} & \text { for } \bar{r} \ll 1, \\
\ln \bar{u}_{g}=-2 \ln \bar{r}+\frac{3}{2} \quad \text { for } \bar{r} \gg 1 .
\end{array}
$$

The change in the slope of $\ln \bar{u}_{g}$ as a function of $\ln \bar{r}$ from $-1 / 2$ at small $\bar{r}$ to -2 at large $\bar{r}$ is evident in Figure 1 of Fukue.

The mass accretion rate can be determined by evaluation at $\bar{r} \gg 1$ since in this limit equation (20) gives

$$
\bar{u}_{g}=\frac{1}{\bar{r}^{2}} \exp \left(-\frac{2}{\bar{r}}+\frac{3}{2}\right) \text { for } \bar{r} \gg 1 \text {. }
$$

Using equation (23) in equation (10) and then evaluating at $r_{\mathrm{gm}}$ gives the Bondi mass accretion rate

$$
\dot{M}_{g}=\pi \frac{\eta \rho_{\mathrm{gm}} M_{*}^{2} G^{2}}{c_{g}^{3}}
$$

where

$$
\eta\left(\epsilon_{\mathrm{BJ}}\right)=\exp \left(-2 \sqrt{\epsilon_{\mathrm{BJ}}}+\frac{3}{2}\right)
$$

is a dimensionless quantity of order unity which occurs repeatedly in the analysis to follow. The radial dependence of the gas mass density in the Bondi regime is given by

$$
\rho_{g}(r)=\frac{\eta \rho_{\mathrm{gm}} M_{*}^{2} G^{2}}{4 r^{2}\left|u_{g}(r)\right| c_{g}^{3}} \text {. }
$$

In the large $r$ limit of the Bondi region $\rho_{g}$ becomes independent of $r$ since in this limit equation (23) shows that $\bar{u}_{g} \sim 1 / \bar{r}^{2}$. This independence permits matching the large $r$ limit of the Bondi region solution to the small $r$ limit of the molecular cloud region solution (given in $\S 3.2$ below) since both these solutions are independent of $r$.
In the small $\bar{r}$ limit, equation (19) gives

$$
\bar{u}_{g}=2 / \bar{r}^{1 / 2},
$$

which corresponds to the free-fall velocity. Combining this with equations (10) and (24) gives the mass density for $r \ll r_{\mathrm{B}}$ to be

$$
\bar{\rho}_{g}(\bar{r})=\frac{\eta}{2 \bar{r}^{3 / 2}} .
$$

The enclosed gas mass at $r_{\mathrm{B}}$ is

$$
\int_{0}^{r_{\mathrm{B}}} 4 \pi r^{2} \rho_{g} d r \simeq 4 \pi \rho_{\mathrm{gm}} r_{\mathrm{B}}^{3} \int_{0}^{1} \bar{r}^{2} \bar{\rho}_{g} d \bar{r}=\frac{4}{3} \pi \eta \rho_{\mathrm{gm}} r_{\mathrm{B}}^{3} .
$$

\subsection{Molecular Cloud Region}

Again ignoring dust mass and dust drag, but now assuming $\bar{r}$ is sufficiently large that $M_{*} \ll \int_{r_{*}}^{r} 4 \pi \xi^{2}\left[\rho_{g}(\xi)\right] d \xi$, equation (8) becomes

$$
\frac{c_{g}^{2}}{\rho_{g}} \frac{d \rho_{g}}{d r}+\frac{G}{r^{2}} \int_{r_{*}}^{r} 4 \pi \xi^{2} \rho_{g}(\xi) d \xi=0
$$

where the subsonic condition $u_{g}^{2} \ll c_{g}^{2}$ has been used. Gas pressure is now balanced by self-gravity rather than by the central object gravity. Equation (30) can be recast in standard form as

$$
\frac{c_{g}^{2}}{4 \pi G} \frac{1}{r^{2}} \frac{d}{d r}\left(\frac{r^{2}}{\rho_{g}} \frac{d \rho_{g}}{d r}\right)+\rho_{g}=0 .
$$

We now define a new dimensionless length suitable for equation (31), namely,

$$
\tilde{r}=\frac{r}{r_{\mathrm{J}}},
$$

where

$$
r_{\mathrm{J}}=\frac{c_{g}}{\sqrt{4 \pi \rho_{\mathrm{gm}} G}}
$$

is the Jeans length.

Equation (31) assumes the dimensionless form

$$
\frac{1}{\tilde{r}^{2}} \frac{d}{d \tilde{r}}\left(\frac{\tilde{r}^{2}}{\bar{\rho}_{g}} \frac{d \bar{\rho}_{g}}{d \tilde{r}}\right)+\bar{\rho}_{g}=0 .
$$

For small $\bar{r}$, we wish to have a solution which is approximately constant in order to be consistent with observations. This constant should be unity in order to connect with the outer limit of the Bondi solution since the outer limit of the Bondi solution has been constructed so as to give $\rho \rightarrow \rho_{\mathrm{gm}}$ for $r=r_{\mathrm{gm}}$. An approximate solution to equation (34) that does this is $\bar{\rho}_{g}=1 /\left(1+\tilde{r}^{2} / 6\right)$, but this solution fails at large $\bar{r}$. However, equation (34) has the exact solution $\bar{\rho}_{g}=2 / \bar{r}^{2}$, which is valid at all $\bar{r}$. Thus, a solution which behaves as $\bar{\rho}_{g}=1 /\left(1+\tilde{r}^{2} / 6\right)$ for small $\tilde{r}$ and as $\bar{\rho}_{g}=2 / \tilde{r}^{2}$ for large $\tilde{r}$ would do the job provided these subsolutions smoothly merge into each other and do not introduce any spurious effects at intermediate values of $\tilde{r}$. The Padé approximation

$$
\bar{\rho}_{g}(\tilde{r})=\left\{1+\frac{\tilde{r}^{2}}{6}\left[1+2\left(\frac{\tilde{r}^{2}}{15+\tilde{r}^{2}}\right)^{2}\right]\right\}^{-1}
$$




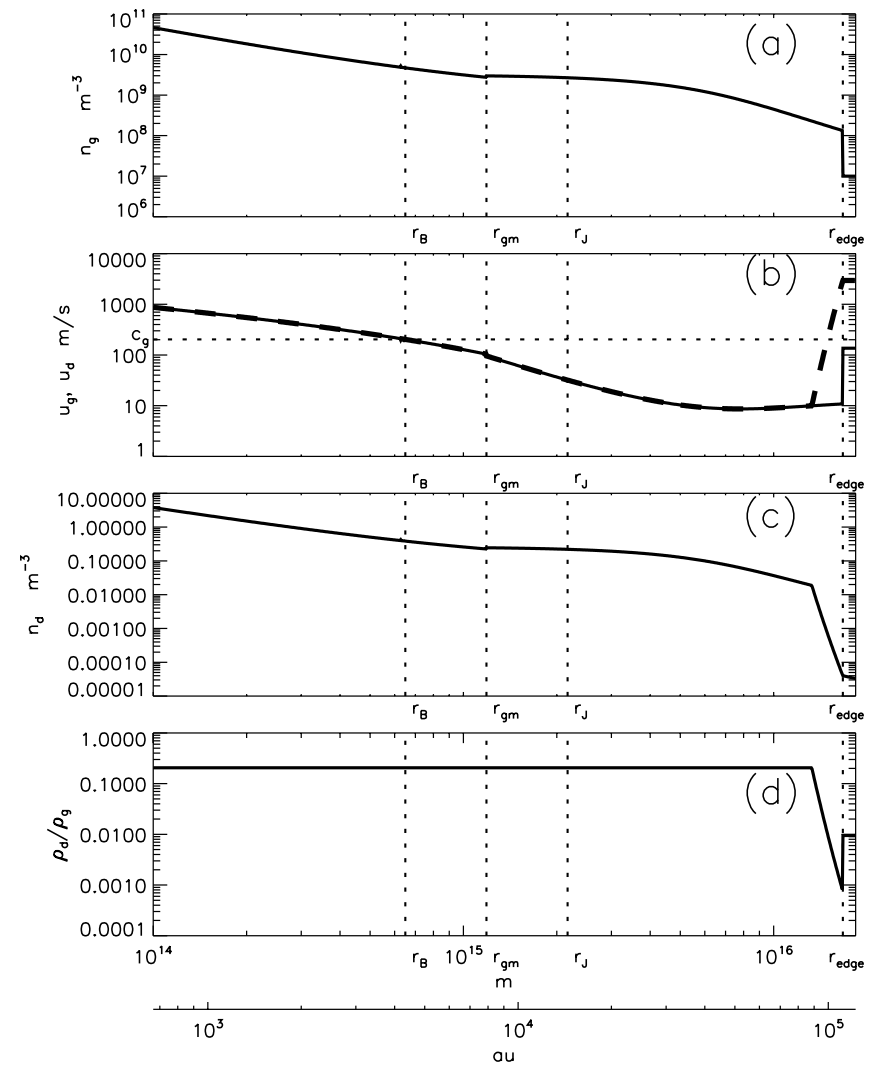

FIG. 1.- Model predictions assuming $\epsilon_{\mathrm{BJ}}=0.3, M_{*}=0.4 M_{\odot}, T_{g}=10 \mathrm{~K}$, $T_{g}^{\mathrm{ISM}}=100 \mathrm{~K}$, and $n_{g}^{\mathrm{ISM}}=10^{7} \mathrm{~m}^{-3}$ : (a) gas density, $(b)$ gas velocity (solid line) and dust velocity (dashed line), (c) dust density, and (d) dust-to-gas mass ratio.

agrees with a direct numerical solution of equation (34) with an error less than $20 \%$ in the range $0<\tilde{r}<9$, an accuracy which is more than adequate for the present analysis. The factor $\left[\tilde{r}^{2} /(15+\right.$ $\left.\left.\tilde{r}^{2}\right)\right]^{2}$ can be thought of as a "switch" which causes $\tilde{r}^{2} / 6$ to become $\tilde{r}^{2} / 2$ at large $\tilde{r}^{2}$.

At $r_{\mathrm{gm}}$, the value of $\tilde{r}$ is $\tilde{r}=r_{\mathrm{gm}} / r_{\mathrm{J}}=\left(\epsilon_{\mathrm{BJ}}\right)^{1 / 2}$ and so equation (34) matches to the Bondi solution at $r_{\mathrm{gm}}$ since by assumption $\epsilon_{\mathrm{BJ}} \ll 1$.

The combined solution spanning the range $0<r<9 r_{\mathrm{J}}$ is therefore

$$
\begin{aligned}
\rho_{g}(r)= & \frac{\eta M_{*}^{2} G^{2}}{4 r^{2}\left|u_{g}(r)\right| c_{g}^{3}} \rho_{\mathrm{gm}} \\
& \text { for } r<r_{\mathrm{gm}}, \\
\rho_{g}(r)= & \rho_{\mathrm{gm}}\left\{1+\frac{r^{2}}{6 r_{\mathrm{J}}^{2}}\left[1+2\left(\frac{r^{2}}{15 r_{\mathrm{J}}^{2}+r^{2}}\right)^{2}\right]\right\}^{-1} \\
& \text { for } r_{\mathrm{gm}}<r<9 r_{\mathrm{J}},
\end{aligned}
$$

where $u_{g}(r)$ is given by equation (20) and both outer and inner solutions converge to $\rho_{\mathrm{gm}}$ at $r=r_{\mathrm{gm}}$. Figure $1 a$ plots $n_{g}(r)=$ $\rho_{g}(r) / m_{g}$ using equation (36) with the assumptions that $\epsilon_{\mathrm{BJ}}=0.3$, $M_{*}=0.4 M_{\odot}, T_{g}=10 \mathrm{~K}, T_{g}^{\mathrm{ISM}}=100 \mathrm{~K}$, and $n_{g}^{\mathrm{ISM}}=10^{7} \mathrm{~m}^{-3}$. The values of $r_{\mathrm{B}}, r_{\mathrm{gm}}$, and $r_{\mathrm{J}}$ are indicated by vertical dashed lines. The solid line in Figure $1 b$ plots the corresponding gas velocity $u_{g}(r)$; note that $u_{g}=c_{g}$ at $r_{\mathrm{B}}$ and that the gas is subsonic to the right of $r_{\mathrm{B}}$.
Specification of $\epsilon_{\mathrm{BJ}}$ determines $\rho_{\mathrm{gm}}$ since substitution of equations (12) and (33) into equation (1) gives

$$
\rho_{\mathrm{gm}}=\epsilon_{\mathrm{BJ}}^{2} \frac{c_{g}^{6}}{\pi M_{*}^{2} G^{3}} .
$$

The separation-of-scales condition is consistent with the assumption $M_{*} \ll \int_{r_{*}}^{r} 4 \pi \xi^{2} \rho_{g}(\xi) d \xi$ for $r \gtrsim r_{\mathrm{J}}$ since

$$
\int_{r_{*}}^{r_{\mathrm{J}}} 4 \pi \xi^{2} \rho_{g}(\xi) d \xi \simeq 4 \pi \rho_{\mathrm{gm}} r_{\mathrm{J}}^{3} / 3=M_{*} / 6 \epsilon_{\mathrm{BJ}}
$$

Equation (37) can be used with equation (29) to calculate the total gas mass enclosed at $r_{\mathrm{B}}$ to be

$$
\int_{0}^{r_{\mathrm{B}}} 4 \pi r^{2} \rho_{g} d r=\frac{\eta}{6} \epsilon_{\mathrm{BJ}}^{2} M_{*},
$$

which is consistent with the Bondi region assumption that $\int_{0}^{r_{\mathrm{B}}} 4 \pi r^{2} \rho_{g} d r \ll M_{*}$.

Equations (24) and (37) show that the gas mass accretion rate can be expressed as

$$
\dot{M}_{g}=\frac{c_{g}^{3}}{G} \epsilon_{\mathrm{BJ}}^{2} \eta .
$$

The gas fluid velocity at $r_{\mathrm{gm}}$ is

$$
u_{g}\left(r_{\mathrm{gm}}\right)=-\frac{\dot{M}_{g}}{4 \pi r_{\mathrm{gm}}^{2} \rho_{\mathrm{gm}}}=-\epsilon_{\mathrm{BJ}} \eta c_{g} .
$$

Bondi accretion will presumably increase $M_{*}$. Because of the existence of jets, not all accreting matter will do this. However, for purposes of estimation, if one assumes that all accreting material causes an increase in $M_{*}$, then $r_{\mathrm{B}}$, which is proportional to $M_{*}$, will increase. On the other hand $r_{\mathrm{J}}$ does not depend on $M_{*}$ and so will remain constant. Thus, $r_{\mathrm{B}} / r_{\mathrm{J}}$ will be a slowly increasing function of time and eventually the presumption that $r_{\mathrm{B}} \ll r_{\mathrm{J}}$ fails.

\subsection{Interface between Molecular Cloud Region and the ISM}

Although gas density and temperature in reality change gradually on entering the cloud from the ISM, for simplicity we assume here that these changes occur in a narrow layer. Integration of equation (4) across this layer while taking into account mass conservation gives continuity of $\rho_{g} u_{g}^{2}+P_{g}$ across the layer, and integration of the mass conservation equation gives continuity of $\rho_{g} u_{g}$ across the layer. This yields Rankine-Hugoniot equations of the form

$$
\begin{gathered}
\bar{n}_{g}\left(r_{\text {edge }}\right)\left\{1+\left[\bar{u}_{g}\left(r_{\text {edge }}\right)\right]^{2}\right\}=\bar{n}_{g}^{\mathrm{ISM}}\left[\frac{T_{g}^{\mathrm{ISM}}}{T_{g}}+\left(\bar{u}_{g}^{\mathrm{ISM}}\right)^{2}\right], \\
\bar{n}_{g}\left(r_{\text {edge }}\right) \bar{u}_{g}\left(r_{\text {edge }}\right)=\bar{n}_{g}^{\mathrm{ISM}} \bar{u}_{g}^{\mathrm{ISM}},
\end{gathered}
$$

where $\bar{n}_{g}^{\mathrm{ISM}}, T_{\mathrm{ISM}}$, and $T_{g}$ are specified and $\bar{u}_{g}^{\mathrm{ISM}}, r_{\text {edge }}, \bar{u}_{g}\left(r_{\text {edge }}\right)$, and $\bar{n}_{g}\left(r_{\text {edge }}\right)$ are to be determined. The bar indicates that densities are normalized to $n_{\mathrm{gm}}$ and velocities are normalized to $c_{g}=$ $\left(\kappa T_{g} / m_{g}\right)^{1 / 2}$. Using equation (42) to substitute for $\bar{n}_{g}\left(r_{\text {edge }}\right) \bar{u}_{g}\left(r_{\text {edge }}\right)$ in equation (41) gives

$\bar{n}_{g}\left(r_{\text {edge }}\right)+\left[\frac{1}{\bar{n}_{g}\left(r_{\text {edge }}\right)}-\frac{1}{\bar{n}_{g}^{\text {ISM }}}\right]\left(\bar{n}_{g}^{\text {ISM }} \bar{u}_{g}^{\text {ISM }}\right)^{2}=\bar{n}_{g}^{\text {ISM }} \frac{T_{g}^{\text {ISM }}}{T_{g}}$. 
However, by using equation (10) evaluated in the ISM at $r_{\text {edge, }}$ equations (39) and (33) give

$$
\bar{n}_{g}^{\mathrm{ISM}} \bar{u}_{g}^{\mathrm{ISM}}=-\eta \epsilon_{\mathrm{BJ}}^{2} \frac{r_{\mathrm{J}}^{2}}{r_{\text {edge }}^{2}},
$$

while equation (35) gives

$$
\bar{n}_{g}\left(r_{\text {edge }}\right) \simeq \frac{2 r_{\mathrm{J}}^{2}}{r_{\text {edge }}^{2}}
$$

so eliminating $r_{\mathrm{J}}^{2} / r_{\text {edge }}^{2}$ between these equations gives

$$
\bar{n}_{g}^{\mathrm{ISM}} \bar{u}_{g}^{\mathrm{ISM}} \simeq-\frac{\eta \epsilon_{\mathrm{BJ}}^{2}}{2} \bar{n}_{g}\left(r_{\text {edge }}\right) .
$$

Substituting for $\bar{n}_{g}^{\text {ISM }} \bar{u}_{g}^{\text {ISM }}$ in equation (43) gives

$$
\begin{aligned}
& -\frac{\left[\bar{n}_{g}\left(r_{\text {edge }}\right)\right]^{2}}{\bar{n}_{g}^{\text {ISM }}}\left(\frac{\eta \epsilon_{\mathrm{BJ}}^{2}}{2}\right)^{2} \\
& \quad+\bar{n}_{g}\left(r_{\text {edge }}\right)\left[1+\left(\frac{\eta \epsilon_{\mathrm{BJ}}^{2}}{2}\right)^{2}\right]-\bar{n}_{g}^{\mathrm{ISM}} \frac{T_{g}^{\mathrm{ISM}}}{T_{g}}=0,
\end{aligned}
$$

a quadratic equation in $\bar{n}_{g}\left(r_{\text {edge }}\right)$. Since $\epsilon_{\mathrm{BJ}}$ is assumed small, an approximate solution to equation (47) can be obtained by balancing the last two terms, in which case

$$
\bar{n}_{g}\left(r_{\text {edge }}\right) \simeq \frac{\bar{n}_{g}^{\mathrm{ISM}}}{\left(1+\eta^{2} \epsilon_{\mathrm{BJ}}^{4} / 4\right)} \frac{T_{g}^{\mathrm{ISM}}}{T_{g}}
$$

Equation (45) then gives

$$
\frac{2 r_{\mathrm{J}}^{2}}{r_{\text {edge }}^{2}}=\frac{\bar{n}_{g}^{\mathrm{ISM}}}{\left(1+\eta^{2} \epsilon_{\mathrm{BJ}}^{4} / 4\right)} \frac{T_{g}^{\mathrm{ISM}}}{T_{g}},
$$

so using equation (33),

$$
r_{\mathrm{edge}} \simeq c_{g}^{2} \sqrt{\frac{1+\epsilon_{\mathrm{BJ}}^{4} \eta^{2} / 4}{2 \pi G n_{g}^{\mathrm{ISM}} \kappa T_{g}^{\mathrm{ISM}}}}
$$

Equation (44) gives

$$
u_{g}^{\mathrm{ISM}}=-\eta \epsilon_{\mathrm{BJ}}^{2} \frac{T_{g}^{\mathrm{ISM}}}{2 T_{g}} \frac{c_{g}}{\left(1+\epsilon_{\mathrm{BJ}}^{4} \eta^{2} / 4\right)},
$$

which combined with equations (42) and (48) gives

\begin{tabular}{|c|c|c|c|}
\hline Parameter & Value (SI base units) & Value (other units) & Reference \\
\hline$r_{\mathrm{B}} \ldots \ldots \ldots \ldots \ldots \ldots$ & $6.5 \times 10^{14} \mathrm{~m}$ & $4.3 \times 10^{3} \mathrm{AU}$ & Eq. (12) \\
\hline$r_{\mathrm{gm}} \ldots \ldots \ldots \ldots \ldots \ldots$ & $1.2 \times 10^{15} \mathrm{~m}$ & $7.9 \times 10^{3} \mathrm{AU}$ & Eq. (2) \\
\hline$r_{\mathrm{J}} \ldots \ldots \ldots \ldots \ldots \ldots \ldots \ldots$ & $2.2 \times 10^{15} \mathrm{~m}$ & $1.4 \times 10^{4} \mathrm{AU}$ & Eq. (33) \\
\hline$n_{\mathrm{gm}} \ldots \ldots \ldots \ldots \ldots \ldots \ldots \ldots \ldots \ldots \ldots \ldots$ & $3.1 \times 10^{9} \mathrm{~m}^{-3}$ & $3.1 \times 10^{3} \mathrm{~cm}^{-3}$ & Eq. (37) \\
\hline$u_{g}\left(r_{\mathrm{gm}}\right) \ldots \ldots \ldots \ldots \ldots$ & $-91 \mathrm{~m} \mathrm{~s}^{-1}$ & & Eq. (40) \\
\hline$c_{g} \ldots \ldots \ldots \ldots \ldots \ldots$ & $2.0 \times 10^{2} \mathrm{~m} \mathrm{~s}^{-1}$ & & Eq. (9) \\
\hline$\dot{M}_{g} \ldots \ldots \ldots \ldots \ldots \ldots \ldots$ & $1.7 \times 10^{16} \mathrm{~kg} \mathrm{~s}^{-1}$ & $2.7 \times 10^{-7} M_{\odot} \mathrm{yr}^{-1}$ & Eq. (39) \\
\hline 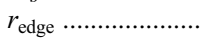 & $1.7 \times 10^{16} \mathrm{~m}$ & $1.1 \times 10^{5} \mathrm{AU}$ & Eq. (50) \\
\hline$r_{\text {edge }} / r_{\mathrm{J}} \ldots \ldots \ldots \ldots \ldots \ldots \ldots$ & 7.7 & & $+2=$ \\
\hline$u_{g}\left(r_{\text {edge }}\right) \ldots \ldots \ldots \ldots$ & $-14 \mathrm{~m} \mathrm{~s}^{-1}$ & & Eq. (52) \\
\hline$u_{g}^{\mathrm{ISM}}$ & $-1.4 \times 10^{2} \mathrm{~m} \mathrm{~s}^{-1}$ & & Eq. (51) \\
\hline$c_{g}^{\mathrm{ISM}} \ldots \ldots \ldots \ldots \ldots \ldots \ldots$ & $6.4 \times 10^{2} \mathrm{~m} \mathrm{~s}^{-1}$ & & Eq. (9) \\
\hline 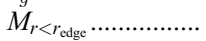 & $2.1 \times 10^{31} \mathrm{~kg}$ & $10 M_{\odot}$ & Eq. (53) \\
\hline 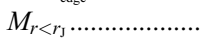 & $4.4 \times 10^{29} \mathrm{~kg}$ & $0.2 M_{\odot}$ & Eq. (54) \\
\hline$M_{r<r_{\mathrm{B}}} \ldots \ldots \ldots \ldots \ldots \ldots$ & $1.8 \times 10^{28} \mathrm{~kg}$ & $0.01 M_{\odot}$ & Eq. (29) \\
\hline
\end{tabular}

$$
u_{g}\left(r_{\text {edge }}\right)=-\frac{\epsilon_{\mathrm{BJ}}^{2} \eta c_{g}}{2} .
$$

The gas mass between $r_{\mathrm{J}}$ and $r_{\text {edge }}$ is

$$
M_{r<r_{\text {edge }}} \simeq \int_{r_{\mathrm{J}}}^{r_{\text {edge }}}\left(\rho_{\mathrm{gm}} 2 \frac{r_{\mathrm{J}}^{2}}{r^{2}}\right) 4 \pi r^{2} d r \simeq 2 c_{g}^{4} \sqrt{\frac{1+\epsilon_{\mathrm{BJ}}^{4} \eta^{2} / 4}{2 \pi G^{3} n_{g}^{\mathrm{ISM}} \kappa T_{g}^{\mathrm{ISM}}}}
$$

TABLE 3

Calculated Quantities for $\epsilon_{\mathrm{BJ}}=0.3, M_{*}=0.4 M_{\odot}, T_{g}=10 \mathrm{~K}$, AND $c_{g}=2 \times 10^{2} \mathrm{~m} \mathrm{~s}^{-1}$

The mass inside the core region of radius $r_{\mathrm{J}}$ is, using equations (35), (33), and (37),

$$
M_{r<r_{\mathrm{J}}} \simeq \rho_{\mathrm{gm}} \int_{r_{\mathrm{gm}}}^{r_{\mathrm{J}}} \frac{4 \pi r^{2}}{1+r^{2} / 6 r_{\mathrm{J}}^{2}} d r \simeq \rho_{\mathrm{gm}} \frac{4 \pi r_{\mathrm{J}}^{3}}{3} \simeq \frac{M_{*}}{6 \epsilon_{\mathrm{BJ}}} .
$$

Table 3 shows quantities predicted by this analysis for $\epsilon_{\mathrm{BJ}}=$ $0.3, M_{*}=0.4 M_{\odot}, T_{g}=10 \mathrm{~K}, T_{g}^{\mathrm{ISM}}=100 \mathrm{~K}$, molecular hydrogen, and $n_{g}^{\text {ISM }}=10^{7} \mathrm{~m}^{-3}$. Note that the gas flow velocities are subsonic (i.e., much smaller than the random or thermal velocity) both in the portion of the cloud external to $r_{\mathrm{B}}$ and in the ISM since in the portion of the cloud external to $r_{\mathrm{B}}$ gas flow velocities $u_{g}$ are much slower than $c_{g}=\left(\kappa T_{g} / m_{g}\right)^{1 / 2}=200 \mathrm{~m} \mathrm{~s}^{-1}$ and in the ISM the gas flow velocity $u_{g}^{\mathrm{ISM}}$ is much slower than $c_{g}^{\mathrm{ISM}}=\left(\kappa T_{g}^{\mathrm{ISM}} / m_{g}\right)^{1 / 2}=640 \mathrm{~m} \mathrm{~s}^{-1}$.

\section{DUST}

\subsection{Overview of Dust}

The mass density of dust in the ISM is well established to be $1 \%$ of the gas mass density (Lilley 1955; Zubko et al. 2004). As mentioned above, it is commonly assumed that this $1 \%$ ratio also holds in a dense molecular cloud. However, Padoan et al. (2006) have recently cast doubt on such an assumption, arguing that if the dust-to-gas mass ratio were indeed $1 \%$ in a dense molecular cloud, then the dust and column densities should have the same spectral power law; i.e., the spatial Fourier power spectra $S(k)$ of dust and gas images should be characterized by the same power law $S(k) \sim k^{-p}$. In fact, Padoan et al. (2006) found that $p$ for the dust differs from $p$ for the gas, indicating a lack of local proportionality between dust and gas.

Takeuchi et al. (2005) have also pointed out that the dust/gas mass ratio in a cloud need not be the same as in the ISM because gas and dust might be subject to different processes. Goldsmith et al. (1997) showed in their Figure 22 that the observed dust-togas ratio varies by an order of magnitude as a function of position in a system of molecular clouds; i.e., the observed dust-to-gas mass ratio in a molecular cloud system is not, as in the ISM, fixed at $1 \%$. As mentioned above, Fukue (2001) presented numerical calculations specifically demonstrating that accretion can enrich the dust-to-gas ratio. As also mentioned above, Fukue (2001) assumed that a Bondi accretion region was directly bounded by the ISM, i.e., did not take into account the molecular cloud scale 
where gas self-gravity is important. This omission resulted in calculated mass accretion rates lower than typical observed values (e.g., Fukue's nominal accretion rate was $M_{g} \sim 10^{-10} M_{\odot} \mathrm{yr}^{-1}$ compared to the observed nominal $\dot{M}_{g} \sim 10^{-9}$ to $10^{-7} M_{\odot}$ rates for pre-main-sequence YSOs reported by Hartmann et al. [1998]). Since gas and dust density scale linearly with accretion rate, the low nominal accretion rate in Fukue's calculation corresponded to a very low dust density and hence optically thin dust. Higher accretion rates give higher dust densities and optically thick dust densities that correspond to the observed opacities of clouds. Nevertheless, Fukue's calculation had the interesting feature of predicting that in certain circumstances the accretion process could cause a 30 -fold increase in the dust-to-gas mass ratio.

We now argue that this enrichment reported by Fukue should be a basic property of dust/gas accretion systems, so the dust/gas mass density ratio in a cloud core should in general be substantially enriched relative to its $1 \%$ ISM ratio. Because the accretion region is now assumed to be bounded by a cloud core rather than by the ISM, this enrichment effect is demonstrated here in association with more realistic mass accretion rates.

Collision properties of gas and dust vary considerably in ISM, cloud, cloud core, and accretion regions. This variation is because the scale lengths are different and because the mean free paths are different. Dust is collisionally decoupled from gas in some regions, but strongly coupled in others. In particular, it turns out that dust is decoupled from gas in the ISM and strongly coupled in the molecular cloud and cloud core, but then can become decoupled again when dust-dust collisions result in dust coagulation. Dust-dust collisionality is closely related to dust opacity since the mean free path for dust-dust collisions is $l_{\mathrm{dd}}=1 / n_{d} \sigma_{d}$, while the distance for unity optical depth is the same if light scattering is geometric, but larger by a factor of $1 / Q_{\text {ext }}$, where the efficiency factor $Q_{\text {ext }}$ accounts for weaker than geometric scattering (i.e., Rayleigh) and for absorption.

Let us start with the ISM. Because of the low gas density in the ISM, dust is collisionally decoupled from gas so if the dust becomes charged, electromagnetic mechanisms can accelerate ISM dust grains to velocities much higher than the $\sim 640 \mathrm{~m} \mathrm{~s}^{-1}$ ISM gas thermal velocity. Meyer et al. (1998), Yan \& Lazarian (2003), Slavin et al. (2004), and Shukla \& Stenflo (2005) have provided examples of such dust acceleration mechanisms.

In contrast, dust is collisionally coupled to gas in molecular clouds because of the high gas density. In cloud cores and/or accretion regions the dust density becomes so high that dust-dust collisions also become important. The condition for dust-dust collisions to be important corresponds approximately to the condition that the dust is optically thick. Thus, if there is an inner region where dust-dust collisions are important, regions external to this inner region will be shielded from central object optical emission and so will not experience any radiation pressure from the central object. Dust-dust collisions result in dust coagulation, a condition where the dust radius $r_{d}$ increases while the overall dust mass density remains invariant. Coagulation has the dual effect of causing the dust to revert to being optically thin and collisionless. Collisionless, coagulated dust exposed to stellar radiation becomes charged via photoemission and so must be described by dusty plasma dynamics rather than by gas dynamics. This dusty plasma regime will be considered in a future publication; preliminary results are presented in Bellan (2007).

\subsection{Dust Grain Size Assumption}

Mathis et al. (1977) reported observations showing that ISM dust grains have a size distribution scaling as $r_{d}^{-3.5}$ with a nominal lower limit radius $r_{\text {low }}=0.005 \mu \mathrm{m}$ and a nominal upper limit $r_{\text {high }}=0.25 \mu \mathrm{m}$. This size distribution is commonly referred to as the MRN dust size distribution. Miyake \& Nakagawa (1993) proposed that the dust size distribution in a circumstellar disk will have larger grains than given by the MRN distribution because in a circumstellar disk where the dust density is high, dust coagulates due to dust-dust collisions. More recently, Przygodda et al. (2003) and van Boekel et al. (2003) have reported direct observational evidence of grain growth in circumstellar disks, while in addition, Dullemond \& Dominik (2005) provided detailed calculations showing a strong tendency for dust grain growth when dust grains collide with each other.

The $r_{d}^{-3.5}$ dust size distribution has important well-known statistical properties. Specifically, an $r_{d}^{-3.5}$ distribution implies (1) most of the mass is contained in the very small number of heavy grains and (2) most of the surface area is contained in the very large number of light grains (Smith \& Dwek 1998). Although the lightest dust grains provide most of the surface area, their radii are so much smaller than visible light wavelengths that their scattering is Rayleigh rather than geometric. Rayleigh scattering is weaker than geometric scattering by a factor of $r_{d}^{4} / \lambda^{4}$, where $\lambda$ is the wavelength of the radiation. Hence, despite being numerous, the very small radius grains are ineffectual at contributing to the optical depth at visible wavelengths, so visible scattering will be due mainly to the larger grains, which also contain most of the mass. Hence, for purposes of both mass inventory and optical depth we consider that dust grains have a nominal radius $r_{d}=0.1 \mu \mathrm{m}$.

\subsection{Dust Velocity in the ISM}

If the velocity of individual dust grains in the ISM were the result of thermal equilibration with gas molecules, the dust kinetic temperature would be the same as the gas molecule kinetic temperature, in which case the random velocity of dust grains in the ISM would be $u_{d}^{\text {ISM }} \sim\left(\kappa T_{g} / m_{d}\right)^{1 / 2}=0.01 \mathrm{~m} \mathrm{~s}^{-1}$. This velocity is so small that there would be insufficient dust flux entering a molecular cloud to populate the cloud with dust in a reasonable time. It has been proposed by several authors that dust attains a much larger random velocity in the ISM because dust is nearly collisionless in the ISM so that collective collisionless mechanisms such as shocks or turbulence could accelerate dust to very large velocities. As mentioned above, Meyer et al. (1998), Yan \& Lazarian (2003), Slavin et al. (2004), and Shukla \& Stenflo (2005) have presented collisionless mechanisms whereby dust grains are accelerated to velocities in the $10 \mathrm{~km} \mathrm{~s}^{-1}$ range.

A typical argument for collisionless acceleration is that a spatially and temporally random magnetic field develops with energy density in thermodynamic equipartition with the gas, i.e., $B^{2} / 2 \mu_{0} \sim n_{g}^{\mathrm{ISM}} \kappa T_{g}^{\mathrm{ISM}}$, so

$$
B \sim \sqrt{2 \mu_{0} n_{g}^{\mathrm{ISM}} \kappa T_{g}^{\mathrm{ISM}}} \sim 2 \times 10^{-10} \mathrm{~T} .
$$

Gradients in this microgauss magnetic field then accelerate charged dust grains via electromagnetic forces so that the dust random kinetic energy becomes comparable to the magnetic energy density, i.e.,

$$
\frac{1}{2} \rho_{d}^{\mathrm{ISM}}\left(U_{d}^{\mathrm{ISM}}\right)^{2} \sim \frac{B^{2}}{2 \mu_{0}},
$$

where $U_{d}^{\mathrm{ISM}}$ is a random velocity that could be pointing in any direction. The nominal random velocity $U_{d}^{\text {ISM }}$ attained by the accelerated grains is thus the dust Alfvén velocity given by 
$v_{\mathrm{A}}^{2}=B^{2} / \mu_{0} \rho_{d}^{\mathrm{ISM}}$. Combining equations (55) and (56) gives the random velocity to be

$$
U_{d}^{\mathrm{ISM}} \simeq \sqrt{\frac{2 \kappa T_{g}^{\mathrm{ISM}}}{m_{g}} \frac{\rho_{g}^{\mathrm{ISM}}}{\rho_{d}^{\mathrm{ISM}}}}
$$

a relationship dependent on the dust-to-gas mass density ratio. Equation (57) gives $U_{d}^{\mathrm{ISM}} \sim 9.3 \mathrm{~km} \mathrm{~s}^{-1}$ for a nominal $1 \%$ ISM dust-to-gas mass ratio and $T_{g}^{\mathrm{ISM}}=100 \mathrm{~K}$. This velocity is 6 orders of magnitude larger than the velocity predicted by thermal equilibration of individual dust grains with gas molecules. Because the dust is assumed to be accelerated by collisionless processes, it will not in general have a Maxwellian distribution, and in fact it would be reasonable to expect that each dust grain has a velocity $\sim U_{d}^{\mathrm{ISM}}$ but with a random direction. This situation would be similar to the neutrons emitted from a fission reaction since such neutrons all have the same kinetic energy but have random velocity directions.

The mean radial velocity of dust grains entering a spherical molecular cloud can be determined by considering the radial velocity component of these dust grains at the surface of the sphere. If $\hat{s}$ denotes the inward normal to the surface at some point on the surface of the sphere, then the radial velocity of a dust grain entering the sphere at this point is $U_{d}^{\mathrm{ISM}} \cos \theta$, where $\theta$ is the angle between the dust grain vector velocity and $\hat{s}$. The angle $\theta$ must lie between $-\pi / 2$ and $\pi / 2$ since dust grains having $\theta$ outside this range will not enter the sphere. Other than being restricted to the range $-\pi / 2<\theta<\pi / 2$, the angle $\theta$ is random. The dust grains have equal probability of having any angle $\theta$ between 0 and $2 \pi$, so the probability of a dust grain angle being between $\theta$ and $\theta+d \theta$ is $d \theta / 2 \pi$. The mean radial velocity of dust grains entering a molecular cloud from the ISM is thus

$$
u_{d}^{\mathrm{ISM}}=\int_{-\pi / 2}^{\pi / 2} U_{d}^{\mathrm{ISM}} \cos \theta \frac{d \theta}{2 \pi}=\frac{1}{\pi} U_{d}^{\mathrm{ISM}} \simeq 3 \mathrm{~km} \mathrm{~s}^{-1} .
$$

The question of whether dust exits a molecular cloud is addressed in the next section.

\subsection{Dust Entrainment by Gas in Molecular Cloud}

The dust equation of motion can be expressed as

$$
\begin{aligned}
u_{d} \frac{d u_{d}}{d r}= & -\frac{G}{r^{2}}\left\{M_{*}+\int_{r_{*}}^{r} 4 \pi \xi^{2}\left[\rho_{g}(\xi)+\rho_{d}(\xi)\right] d \xi\right\} \\
& -\left(u_{d}-u_{g}\right) \frac{\rho_{g}}{m_{d}} \sigma_{d} \sqrt{c_{g}^{2}+\left(u_{d}-u_{g}\right)^{2}}
\end{aligned}
$$

where it is recalled that both $u_{d}$ and $u_{g}$ are negative, corresponding to radial inward motion.

We wish to investigate the possibility that $\rho_{d} / \rho_{g}$ could increase from its 1\% ISM value as dust and gas enter the cloud, core, and Bondi accretion region. However, we assume that $\rho_{d} / \rho_{g}$ remains sufficiently small compared to unity that the back-reaction of dust on gas dynamics can be ignored. Thus, $u_{g}(r)$ and $\rho_{g}(r)$ are assumed to remain as discussed in $\S \S 3.1$ and 3.2. Dust enters the cloud from the ISM with a velocity much higher than the gas thermal and fluid velocities but then collides with the gas, thereby slowing down until moving at nearly the same inward fluid velocity as the gas. Collisions thus cause the dust to become entrained by the gas inflow (Fukue 2001).

The collisional slowing down can be estimated by assuming that the gravitational force term in equation (59) can be ignored because of the high dust velocity. Furthermore, $u_{d}$ is much larger than both $u_{g}$ and $c_{g}$ when the dust enters the cloud from the ISM. In this case and using

$$
\frac{\sigma_{d}}{m_{d}}=\frac{3}{4 r_{d} \rho_{d}^{\mathrm{int}}}
$$

where $\rho_{d}^{\text {int }} \simeq 2 \times 10^{3} \mathrm{~kg} \mathrm{~m}^{-3}$ is the intrinsic mass density of the dust, equation (59) becomes

$$
\frac{d u_{d}}{d r}=-u_{d} \frac{3 \rho_{g}(r)}{4 r_{d} \rho_{d}^{\text {int }}} .
$$

Integration of equation (61) starting from the cloud edge and going inward gives

$$
u_{d}(r)=u_{d}\left(r_{\text {edge }}\right) \exp \left[-\int_{r}^{r_{\text {edge }}} \frac{3 \rho_{g}(\xi)}{4 r_{d} \rho_{d}^{\text {int }}} d \xi\right] .
$$

The dashed line in Figure $1 b$ shows the dust velocity given by equation (62) for the gas density given in Figure $1 a$ and assuming a $3 \mathrm{~km} \mathrm{~s}^{-1}$ dust mean radial entrance velocity. It is seen that the dust rapidly slows down until it achieves the same velocity as the gas, at which point it is assumed to be entrained by the gas. Dust will thus become entrained by gas at a radius $r_{\text {ent }}$, where $r_{\text {ent }}$ satisfies

$$
u_{d}^{\text {ISM }} \exp \left[-\int_{r_{\text {ent }}}^{r_{\text {edge }}} \frac{3 \rho_{g}(r)}{4 r_{d} \rho_{d}^{\text {int }}} d r\right] \sim u_{g}^{\text {edge }} \sim \frac{c_{g}}{20}
$$

Using equation (58) to estimate $u_{d}^{\text {ISM }}$, equation (63) becomes

$$
\int_{r_{\mathrm{ent}}}^{r_{\text {edge }}} \frac{3 \rho_{g}(r)}{4 r_{d} \rho_{d}^{\text {int }}} d r \sim \ln \left(\frac{20 u_{d}^{\text {ISM }}}{c_{g}}\right) \simeq 5.7 .
$$

If the gas density in the $r \gg r_{\mathrm{J}}$ portion of the cloud is sufficiently large to provide entrainment, then $\rho_{g}(r)$ can be approximated in this region as $\rho_{g}(r) \simeq 2 \rho_{\mathrm{gm}} r_{\mathrm{J}}^{2} / r^{2}$, so equation (64) becomes

$$
\frac{3 \rho_{\mathrm{gm}} r_{\mathrm{J}}}{2 r_{d} \rho_{d}^{\text {int }}}\left(\frac{r_{\mathrm{J}}}{r_{\text {ent }}}-\frac{r_{\mathrm{J}}}{r_{\text {edge }}}\right) \simeq 5.7 .
$$

Table 3 gives $r_{\text {edge }} / r_{\mathrm{J}} \simeq 7.7$, and since $3 \rho_{\mathrm{gm}} r_{\mathrm{J}} / 2 \rho_{d}^{\text {int }} r_{d}=1.7 \times$ $10^{2}$ this gives $r_{\mathrm{ent}} \simeq 6 r_{\mathrm{J}}$. As assumed, the dust becomes entrained by the gas at a location in the $r \gg r_{\mathrm{J}}$ portion of the cloud.

The entrainment of dust by gas means that the radial flow of dust from the ISM to the cloud is one-way inward; i.e., there is no radial outward flow of individual dust grains from the cloud back to the ISM. This one-way inward behavior of dust grains is in contrast to gas. Because gas molecules collide with each other, some gas molecules have inward radial velocities and some have outward radial velocities. However, there are more inward than outward moving gas molecules, and the net inward gas fluid velocity at $r_{\text {edge }}$ is a consequence of this difference. The situation is somewhat analogous to a home vacuum cleaner ingesting macroscopic particles and air; individual air molecules in the vacuum hose have inward or outward velocities with magnitude of the order of the air thermal velocity, the mean air velocity in the hose is inward and much slower than the air thermal velocity, and all the macroscopic particles in the hose have inward velocities. 


\section{two-lane highway merging one-lane highway}

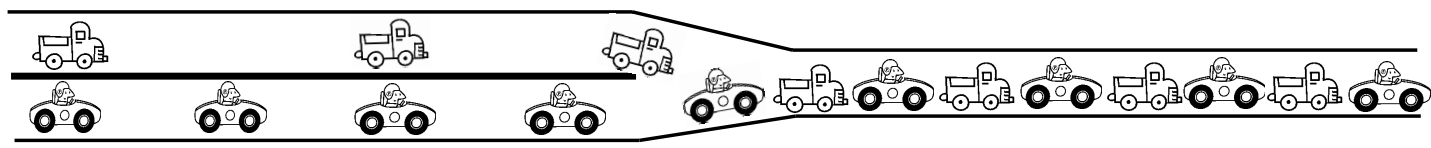

\section{truck speed twice car speed truck density half car density \\ truck speed same as car speed truck density enriched $2 \mathrm{x}$}

FIG. 2.-Because trucks entering the one-lane segment slow down whereas cars do not, the truck-to-car density ratio is enriched in the one-lane segment compared to the density ratio in the two-lane segment.

\subsection{Enrichment of Dust-to-Gas Mass Ratio}

Equations (10) and (11) can be evaluated at any radius. Evaluating at $r_{\text {edge }}$ and at $r_{\text {gm }}$ gives

$$
\begin{aligned}
& 4 \pi r_{\text {edge }}^{2} \rho_{g}^{\text {ISM }} u_{g}^{\text {ISM }}=4 \pi r_{\mathrm{gm}}^{2} \rho_{g}\left(r_{\mathrm{gm}}\right) u_{g}\left(r_{\mathrm{gm}}\right), \\
& 4 \pi r_{\text {edge }}^{2} \rho_{d}^{\mathrm{ISM}} u_{d}^{\mathrm{ISM}}=4 \pi r_{\mathrm{gm}}^{2} \rho_{d}\left(r_{\mathrm{gm}}\right) u_{d}\left(r_{\mathrm{gm}}\right) .
\end{aligned}
$$

Dividing equation (66) by equation (67) gives

$$
\frac{\rho_{d}\left(r_{\mathrm{gm}}\right) / \rho_{g}\left(r_{\mathrm{gm}}\right)}{\rho_{d}^{\mathrm{ISM}} / \rho_{g}^{\mathrm{ISM}}}=\frac{u_{d}^{\mathrm{ISM}} / u_{g}^{\mathrm{ISM}}}{u_{d}\left(r_{\mathrm{gm}}\right) / u_{g}\left(r_{\mathrm{gm}}\right)} .
$$

Since the dust is certainly fully entrained by the time it reaches $r_{\mathrm{gm}}$ we can assume $u_{d}\left(r_{\mathrm{gm}}\right) / u_{g}\left(r_{\mathrm{gm}}\right)=1$. Using equation (51) to give $u_{g}^{\text {ISM }}$ and equations (57) and (58) to give $u_{d}^{\text {ISM }}$, equation (68) becomes

$$
\frac{\rho_{d}\left(r_{\mathrm{gm}}\right)}{\rho_{g}\left(r_{\mathrm{gm}}\right)}=\frac{2+\epsilon_{\mathrm{BJ}}^{4} \eta^{2} / 2}{\pi \eta \epsilon_{\mathrm{BJ}}^{2}} \sqrt{\frac{2 T_{g}}{T_{g}^{\mathrm{ISM}} \frac{\rho_{d}^{\mathrm{ISM}}}{\rho_{g}^{\mathrm{ISM}}}}}
$$

Figure $1 c$ shows the dust density and Figure $1 d$ shows the dustto-gas mass ratio as function of radius, showing that enrichment occurs so that the dust-to-gas ratio increases from its 0.01 ISM value to become somewhat less than unity. For the example parameters in Table 3 and a 1\% ISM dust-to-gas mass ratio, equation (69) predicts $\rho_{d}\left(r_{\mathrm{gm}}\right) / \rho_{g}\left(r_{\mathrm{gm}}\right)=0.2$ for $\epsilon_{\mathrm{BJ}}=0.3, T_{g}=10 \mathrm{~K}$, and $T^{\mathrm{ISM}}=100 \mathrm{~K}$; this corresponds to the saturated value in Figure $1 d$ and represents a 20 -fold enrichment of the dust-to-gas mass ratio over its ISM value.

An everyday example of the enrichment effect predicted by equation (68) is where traffic on a two-lane highway merges to flow on a one-lane highway as sketched in Figure 2. In the twolane segment $(l e f t)$, the trucks move at twice the speed of the cars and have half the density of the cars. The cars are assumed to have the same speed in the two-lane and one-lane highway segments; i.e., the cars do not slow down. The trucks have to slow down when they enter the one-lane segment because in the one-lane segment the trucks must go at the same speed as the cars. Slowing down by a factor of 2 causes the number of trucks per length of road to double because in steady state the flux of trucks (product of the truck speed and the trucks per length of road) must be the same in the one- and two-lane segments. The density of trucks to cars is thus enriched by a factor of 2 in the one-lane highway segment compared to its value in the two-lane highway segment.

\subsection{Radiation Pressure and Optical Depth}

Abbas et al. (2003) used a calibrated electrodynamic balance to measure the radiation pressure force exerted on a $r_{d}=0.125 \mu \mathrm{m}$ particle by a $0.532 \mathrm{~nm}$ wavelength laser. They found that the force exerted by a laser intensity of $8 \times 10^{5} \mathrm{~W} \mathrm{~m}^{-2}$ was $3 \times 10^{-17} \mathrm{~N}$. If such a particle were in the gravitational field of a star with luminosity $L_{*}$, the radiation pressure force on the particle would thus be

$$
F_{\mathrm{rad}}=\frac{L_{*} e^{-\tau(r)}}{4 \pi r^{2}} \frac{3 \times 10^{-17}}{8 \times 10^{5}} \mathrm{~N}
$$

where

$$
\tau(r)=\int_{r_{*}}^{r} Q_{\mathrm{ext}} n_{d}(\xi) \sigma_{d} d \xi
$$

is the optical depth for light traveling from the star to radius $r$ and $Q_{\text {ext }}$ is the extinction efficiency. The gravitational force would be

$$
F_{g}=\frac{m_{g} M_{*} G}{r^{2}} \mathrm{~N},
$$

so the ratio would be

$$
\frac{F_{\mathrm{rad}}}{F_{g}}=\frac{L_{*} e^{-\tau(r)}}{4 \pi} \frac{3 \times 10^{-17}}{8 \times 10^{5}} \frac{1}{m_{g} M_{*} G} .
$$

Assuming $m_{g}=10^{-17} \mathrm{~kg}$ for a nominal $r_{d}=10^{-7} \mathrm{~m}$ dust grain gives

$$
\frac{F_{\mathrm{rad}}}{F_{g}}=0.9 e^{-\tau(r)} \frac{L_{*} / L_{\odot}}{M_{*} / M_{\odot}},
$$

so a dust grain could in principle be subject to significant radiation force if $\tau \ll 1$ as was proposed by Fukue. Table 1 of Abbas et al. (2003) gives the calculated radiation pressure efficiency $Q_{\mathrm{pr}}=0.28$ for a $r_{d}=0.125 \mu \mathrm{m}$ dust grain illuminated by $532 \mathrm{~nm}$ light. This is in reasonable agreement with their measured value $Q_{\mathrm{pr}}=0.22$; Abbas et al. (2003) also presented a calculated extinction efficiency $Q_{\text {ext }}=0.33$ and a calculated scattering efficiency $Q_{\text {sca }}=0.16$ that together would be in reasonable agreement with the measured $Q_{\mathrm{pr}}$. Figure $1 c$ shows that dust for the representative parameters discussed here has a density $n_{d} \simeq 1 \mathrm{~m}^{-3}$ at $r_{\mathrm{B}}$ and that this density increases for $r<r_{\mathrm{B}}$. Table 3 gives $r_{\mathrm{B}}=6.5 \times 10^{14} \mathrm{~m}$. Thus, a lower bound for the optical depth at $r_{\mathrm{B}}$ can be estimated using

$\tau\left(r_{\mathrm{B}}\right)>Q_{\mathrm{ext}} n_{d}\left(r_{\mathrm{B}}\right) \sigma_{d} r_{\mathrm{B}}=0.33 \pi\left(10^{-7}\right)^{2}\left(6.5 \times 10^{14}\right)=6.7$. 
Starlight would thus be attenuated by a factor greater than $\exp (-6.7) \simeq 10^{-3}$ and so optical radiation from the star should be fully extinguished by the time it reaches $r_{\mathrm{B}}$, in which case there would be no significant radiation pressure on dust at radii of the order of $r_{\mathrm{B}}$ or larger. The reasons why we differ from Fukue by concluding that radiation pressure is unimportant are as follows: (1) Fukue's adiabatic assumption resulted in gas that was 1600 times hotter at small $r$ so for a given pressure the gas density would be 1600 times less, and so for a given dust-to-gas ratio, the dust density would be much lower; (2) Fukue also used a much lower value of $Q_{\text {ext }}$ than the value given in Abbas et al. (2003).

\section{DISCUSSION}

The logical requirement that the Bondi length must be smaller than the Jeans length introduces the dimensionless parameter $\epsilon_{\mathrm{BJ}}$, given by the ratio of these lengths. An intermediate distance $r_{\mathrm{gm}}$ can be defined as the geometric mean of the Bondi and Jeans lengths, so if the two lengths are well separated, $r_{\mathrm{gm}}$ is much larger than the Bondi length but much smaller than the Jeans length. Thus, $r_{\mathrm{gm}}$ constitutes the large $r$ limit of the solution to the Bondi problem and the small $r$ limit of the solution to the Jeans problem. Matching the Bondi and Jeans solutions at $r_{\mathrm{gm}}$ provides a solution valid over scales ranging from smaller than the Bondi length to larger than the Jeans length. This accounts for transonic flow, mass accretion, and gravitational self-confinement in one self-consistent solution to the gas equation.
By assuming that the dust mass density and energy density are not larger than the corresponding gas densities, the gas equations can be evaluated ignoring interaction with dust. Once the gas behavior has been worked out, the dust can be considered as moving through a predetermined gas profile that retards the dust due to frictional drag. The dust slows down greatly whereas the gas velocity changes relatively little, a distinction that enriches the dust-to-gas mass ratio. Plausible values of $\epsilon_{\mathrm{BJ}}$ suggest that the dust-to-gas mass ratio will always be enriched to be somewhat less than unity, because at unity the assumption that the gas is unaffected by dust fails. The high dust density at small radius means that dust-dust collisions will become important and cause coagulation of dust. This coagulation will cause the dust to become optically thin and collisionless again. Because the coagulated dust is optically thin, it will absorb photons from the star and become electrically charged due to photoemission of electrons. Two-dimensional motion of collisionless, charged dust in a gravitational field resulting in a poloidal field dynamo has been discussed in Bellan (2007); three-dimensional motion and resulting poloidal/toroidal field dynamo action will be discussed in a future publication.

The author wishes to thank an anonymous referee for thoughtful comments.
Abbas, M. M., et al. 2003, J. Geophys. Res. Space Phys., 108 (A6), 1229

Bellan, P. M. 2007, Phys. Plasmas, 14, 122901

Bellan, P. M., You, S., \& Hsu, S. C. 2005, Ap\&SS, 298, 203

Bondi, H. 1952, MNRAS, 112, 195

Bonnor, W. B. 1956, MNRAS, 116, 351

Dullemond, C. P., \& Dominik, C. 2005, A\&A, 434, 971

Ebert, R. 1955, Z. Astrophys., 37, 217

Evans, N. J., Rawlings, J. M. C., Shirley, Y. L., \& Mundy, L. G. 2001, ApJ, 557,193

Fukue, J. 2001, PASJ, 53, 275

Goldsmith, P. F., Bergin, E. A., \& Lis, D. C. 1997, ApJ, 491, 615

Hartmann, L., Calvet, N., Gullbring, E., \& D'Alessio, P. 1998, ApJ, 495, 385

Hsu, S. C., \& Bellan, P. M. 2002, MNRAS, 334, 257

Lamers, H. J. G. L. M., \& Cassinelli, J. P. 1999, Introduction to Stellar Winds (Cambridge: Cambridge Univ. Press)

Lilley, A. E. 1955, ApJ, 121, 559

\section{REFERENCES}

Mathis, J. S., Rumpl, W., \& Nordsieck, K. H. 1977, ApJ, 217, 425

Meyer, J. P., Drury, L. O., \& Ellison, D. C. 1998, Space Sci. Rev., 86, 179

Miyake, K., \& Nakagawa, Y. 1993, Icarus, 106, 20

Padoan, P., Cambresy, L., Juvela, M., Kritsuk, A., Langer, W. D., \& Norman, M. L. 2006, ApJ, 649, 807

Przygodda, F., van Boekel, R., Abraham, P., Melnikov, S. Y., Waters, L. B. F. M., \& Leinert, C. 2003, A\&A, 412, L43

Shukla, P. K., \& Stenflo, L. 2005, ApJ, 629, L93

Slavin, J. D., Jones, A. P., \& Tielens, A. G. G. M. 2004, ApJ, 614, 796

Smith, R. K., \& Dwek, E. 1998, ApJ, 503, 831

Takeuchi, T., Clarke, C. J., \& Lin, D. N. C. 2005, ApJ, 627, 286

van Boekel, R., Waters, L. B. F. M., Dominik, C., Bouwman, J., de Koter, A., Dullemond, C. P., \& Paresce, F. 2003, A\&A, 400, L21

Yan, H., \& Lazarian, A. 2003, ApJ, 592, L33

Zubko, V., Dwek, E., \& Arendt, R. G. 2004, ApJS, 152, 211 